\title{
Passing the Red Pen: EvoS Journal Offers an Early Education in Peer Review
}

\author{
Rosemarie Sokol Chang
}

Published online: 20 February 2011

(C) Springer Science+Business Media, LLC 2011

\begin{abstract}
EvoS Journal: The Journal of the Evolutionary Studies Consortium is the open-access, peer-reviewed journal borne of the EvoS (Evolutionary Studies) Consortium movement. The journal has two separate, yet related goals: to publish scholarly articles regarding the intersection of higher education and evolutionary theory, and to publish articles written and reviewed by undergraduates that incorporate an evolutionary perspective. Undergraduates who publish and review for EvoS Journal get the chance to see their work published alongside that of experts within the field of evolutionary studies, and to see the interdisciplinary and widespread applications of evolutionary theory to human life and beyond. The current study examines whether the interdisciplinary nature of EvoS Journal and its initiation of students into the peer-review process set EvoS Journal apart from more traditional undergraduate journals. EvoS Journal was compared to other journals listed by the Council for Undergraduate Research. While EvoS Journal mirrors many traditional undergraduate journals in their employment of student reviewers and publishing work originating from any U.S. institution, it differs by both its interdisciplinary focus and by serving as an outlet for both student and professional publications. The implications are discussed in terms of the benefits of interdisciplinary and peer-review training to students in the classroom, including improvements in writing, interpersonal relations, and real-world skills.
\end{abstract}

Keywords EvoS Journal - Undergraduate peer review . Evolutionary Studies $\cdot$ Interdisciplinary journal

\section{R. S. Chang $(\bowtie)$}

Department of Psychology,

State University of New York at New Paltz,

New Paltz, NY 12561, USA

e-mail: rischang@gmail.com
EvoS Journal: The Journal of the Evolutionary Studies Consortium (EvoS Journal, http://evostudies.org/evosjournal/about-the-journal/) is an outlet for professionals to publish work regarding evolutionary theory in higher education, and for undergraduates to publish research, theoretical, and review articles that incorporate an evolutionary perspective. Both of these goals underscore a few major aspects of the Evolutionary Studies (EvoS) Consortium: the importance of evolutionary theory to interdisciplinary scholarship and encouraging undergraduates to engage in the scientific process.

Evolutionary science lends itself to interdisciplinary scholarship due to the applicability of evolutionary theory across organisms, and to aspects within an organism. For example, the theory of evolution has been used to explain much of human life, from physical features, behavior, and emotions to culture and medical symptoms. Garcia et al. (2011) have shown for the study of human behavior that publications from journals that focus on the evolutionary approach have articles first-authored by scholar's from a wider spread of disciplines than publications from a leading psychology journals that do not incorporate evolution specifically. The Evolutionary Studies Consortium itself is evidence of the interdisciplinary nature of evolutionary theory, having contact members from eight disciplines, as well as course listings in eight different disciplines just among the first two schools with evolutionary studies programs (i.e., Binghamton University and SUNY New Paltz). Perhaps more compelling are the returns in factual knowledge, critical thinking, and ability to recognize the applications of evolutionary theory to humans garnered by students from instruction on evolutionary theory (O'Brien et al. 2009).

The second goal of EvoS Journal, to engage undergraduates in the scientific process, is achieved by publishing undergraduate-authored manuscripts that have been peer 
reviewed by undergraduate editorial board members. Since its inception in 2009, EvoS Journal has published 11 works, four of which were first-authored by undergraduates. Further, EvoS Journal has received 17 submissions from undergraduates since launching its call for papers, employing multiple undergraduate reviewers along the way.

The benefits of peer review to undergraduate students have been well noted from studies within the classroom. Assignments that mimic the academic peer-review process have resulted in an improved understanding of the scientific publishing process, and increased final draft grades from previous semesters (Guilford 2001). Further, students have noted improvement in writing and a greater understanding of their own strengths and weakness after learning how to critique the work of peers (Liu et al. 2002). Assignments that require students to respond to early drafts written by others results in writing and thinking improvements (Dominick et al. 1997; Lundstrom and Baker 2009; Newell 1998; Paulus 1999; Sargent 1997), interpersonal relationship skills such as communication and cooperation (Dochy et al. 1999; Liu et al. 2002; McGourty et al. 1998; Silva and Moreira 2003), and problem solving and organizational skills (McGourty et al. 1998; Newell 1998; Sargent 1997). These benefits are only attained with proper instruction for critiquing the writing of another student. EvoS Journal employs a framework for instruction in a couple of ways.

The process of review for EvoS Journal involves hosting a traditional editorial board for manuscripts authored by professionals, and a board made up of undergraduate editorial board members (http://evostudies.org/evos-journal/ editorial-board/). The journal enlists some faculty editorial board members to serve as faculty advisors to the undergraduates. These faculty advisors represent different academic disciplines, and nominate undergraduates from within their disciplines to serve as editorial board members. The faculty advisor then oversees the review process, advising students who write the reviews. Thus, these advisors can assist when reviewing statistics or advanced topics to which the undergraduate reviewers have not yet been exposed. In addition to faculty advisors, undergraduate editorial board members have at their disposal detailed review instructions, including a sample review (http://evostudies. org/pdf/EvoSUnderReviewers.pdf).

Given the benefits to undergraduates of the interdisciplinarity of evolutionary theory and instruction in peer review, EvoS Journal has laudable goals, which presumably offer the chance for students to improve their writing and thinking and interpersonal and organizational skills outside of the classroom. EvoS Journal offers undergraduates the chance to engage in the peer-review process, and encourages undergraduates to submit interdisciplinary scholarship for review. However, EvoS Journal is not alone in publishing manuscripts authored and reviewed by undergraduate students. For example, the Council on Undergraduate Research (CUR) lists 62 electronic undergraduate journals on its website (http://www.cur.org/ ugjournal.html). Thus, to establish how unique EvoS Journal is in regards to interdisciplinarity and peer review, in the following analysis, EvoS Journal is compared with these CUR listed journals in two ways:

1. The general interdisciplinarity of undergraduate journals

2. Whether undergraduates are enlisted to review submissions

In addition, as a measure of where EvoS Journal lies in respect to the promotion of student-faculty interaction, and cross-institution scholarship, EvoS Journal is compared with the CUR listed journals as follows:

1. Whether the journals publish works by faculty as well as students

2. Whether submissions are limited to students at a particular institution

\section{Methods}

The journals listed by the Council on Undergraduate Research serve as the basis of comparison in these analyses. Out of the 62 journals listed, only 57 could be located online. For some journals, the links were broken and the journals unable to be located through a search engine. Out of these remaining 57 journals, which includes EvoS Journal, the following information was recorded: from which disciplines authors are invited to submit, whether undergraduates serve as reviewers, whether or not faculty publish in the journal, and whether submissions are limited to a specific institution or a consortium of schools. This information served as the basis for chi-square analyses comparing the different aspects of undergraduate journals.

\section{Results}

Fifty-two journals had information regarding whether they were interdisciplinary (i.e., papers from any disciplinary focus are welcome) or discipline specific (i.e., submissions are restricted to one discipline, e.g., mathematics; or a few disciplines, e.g., humanities.) Significantly more journals are discipline specific $(N=37)$ than interdisciplinary $(N=19$; $\left.X^{2}(1, N=52)=5.78, p=0.016\right)$.

Forty-seven journals had clearly stated editorial board members. Out of these journals, there was a significant difference among the three categories created: journals that employ only undergraduates as reviewers $(N=25)$, journals that employ only faculty as reviewers $(N=15)$, and journals 
Table 1 Undergraduate reviewer open-ended responses to the question "What have you learned by serving as an editorial board member for EvoS Journal?," arranged thematically

Review process/larger community

Interdisciplinarity/application of evolutionary theory

1. I learned more about the topic of the paper I reviewed. I also gained a better understanding of the review process.

2. Serving as an editorial board member has provided me with the opportunity to influence my peers in the academic community. This position has also allowed me to broaden my own interests in evolutionary psychology, as well as be exposed to new perspectives.
3. People have different writing styles; I did learn more about writing for an evolutionary journal as I have never written for one. For example, the constant reflecting on evolutionary theory and tying it in to newer ideas is very interesting; it is important to back up your statements and know the theory to draw rational or reasonable conclusions.

4. I have learned more about writing style and the diverse applications of evolutionary theory to psychology than anything else.

5. I have learned to be more critical about my own writing and to review a significant amount of supporting articles.
4. I have learned more about writing style and the diverse applications of evolutionary theory to psychology than anything else.

6. I have learned that evolutionary theory is steadily being integrated with the subdivisions of psychology.

Numbers identify student responses, and thus might appear more than once if a response fit more than one category

that employ both undergraduates and faculty as reviewers $\left(N=7 ; X^{2}(2, N=47)=10.38, p=0.005\right)$. A separate chi-square analysis compared only those journals reviewed by undergraduates $(N=25)$ with those journals reviewed by both faculty and undergraduates $(N=7)$, between which there was a significant difference $\left(X^{2}(1, N=32)=10.13, p=0.002\right)$.

Fifty-seven schools had information regarding whether faculty were invited to publish in the journals as well as undergraduates. There was a significant difference between the number of journals that published undergraduateauthored work only $(N=52)$ and journals that publish undergraduate- and faculty-authored work $\left(N=5 ; X^{2}(1\right.$, $N=57)=38.75, p<0.0001)$.
Fifty-five journals clearly stated whether submissions were limited to students from a particular institution or a region of schools $(N=32)$ or open to students from any institution $(N=23)$. There was no difference between the two categories $\left(X^{2}(1, N=55)=1.47, p=0.22\right)$.

\section{Discussion}

While EvoS Journal is similar to many undergraduate journals in its use of undergraduates in the peer-review process, and accepting submissions from students at any institution, it differs both in its interdisciplinary focus, and by

Table 2 Undergraduate reviewer open-ended responses to the question "What are the major academic benefits to serving as an editorial board member for EvoS Journal?," arranged thematically

Review process/larger community

3. Allows you to help contribute to your peers and to the field, heightens your own editorial abilities when reviewing your own work, feel like an active member in the community.4. First, the opportunity to view the work of peers from other universities has been enlightening. Second, I have gained more experience in the blind peer-review process which is common in the scientific/ academic world.

5. Increasing my exposure to academic articles and novel ideas.
Writing improvements

1. You get to think critically and express your opinion.

2. Serving as a board member helped to sharpen my analytical and critical reading skills. I think this opportunity definitely provided me with some fundamental building blocks to help me be more successful at evaluating my own work as well as my peers, upon which I can continue to build throughout my career in academia.

3. Gives you an example of how others approach the research and writing process, allows you to explore how you may have done things differently.

3. Gives you an example of how others approach the research and writing process, allows you to explore how you may have done things differently.

6. It is an opportunity to improve your critical thinking skills to more effectively evaluate and prepare your own research.

Student responses are denoted by numbers that match with the responses in Table 1. Portions of a response might appear more than once if a response fit more than one category 
publishing work authored and reviewed by both faculty and undergraduate students. The two main goals of EvoS Journal, to foster interdisciplinary scholarship and to engage students in the scientific process, are promoted by the journal, though the latter is not unique among the journals listed by the Council for Undergraduate Research. Arguably, choosing a sample from the CUR can only provide a limited scope, as they are necessarily related to undergraduate scholarship. A more revealing study would be a comparison to all academic journals in the United States, a sample that is beyond the scope of the current study.

The interdisciplinarity of EvoS Journal is reflected among the editorial board, which consists of members from ten distinct disciplines, and in the publications authored by undergraduates. These incorporate evolutionary theory (a traditionally biological theory) with topics more traditionally central to other disciplines, for example education and psychology (Muller 2010) and a multidisciplinary approach to the study of religion (Smith and Arrow 2010).

The benefits of faculty-student collaboration are noteworthy, in terms of student and faculty retention, student learning, and fostering a personal connection with faculty mentors (see Elgren and Hensel 2006 for a review). By publishing undergraduate-authored scholarship alongside faculty-authored scholarship, EvoS Journal hopes to echo these benefits from curriculum and traditional educational experiences, and improve the connection students feel to the greater scientific community.

While the benefits of the peer-review process to undergraduates can be inferred from studies in the classroom, actual tests of the benefits of professional peer review are needed. The editor of EvoS Journal conducted an online, anonymous survey of the undergraduate editorial board members. They reflect the benefits of peer review in their experiences in reviewing (see Tables 1 and 2). These comments reflect improvements in writing, understanding of the scientific peer-review process, and reflection on the interdisciplinary nature of evolutionary theory. However, more formal tests of pre- and post-ability in writing, interpersonal skills, and problem-solving skills are needed for undergraduate-reviewed journals.

\section{Conclusions}

EvoS Journal offers an important interchange between interdisciplinary scholarship and collaboration between faculty and students. There may be some question as to whether the benefits go both ways, in other words is EvoS Journal improved by having undergraduate editorial board members involved? The editor would confirm the double-sided benefits, noting the detail and thoughtfulness in student reviews. A stronger test would set undergraduate reviews alongside faculty reviews, and have faculty members rate the reviews in terms of the comprehensiveness of the critiques. ${ }^{1}$ Certainly such a test is a feasible future direction.

A second possible advantage of undergraduate reviewers is that they are not yet connected to the undergraduates authoring papers and stand to write a less biased review than faculty reviewers. In a study of articles published in widely read psychology journals that practiced non-blind review, 12 previously published articles were resubmitted with false author names and affiliations (Peters and Ceci 1982). Of these 12, eight were rejected from the journals, many on the grounds of "serious methodological flaws."

A disadvantage of using undergraduates for peer review is the turnover rate of student reviewers. Since reviewing a manuscript requires some background training, students usually join the editorial board in their junior or senior year of college. Thus, the time of service is between one and two years. This disadvantage is surely to the advantage of the greater scientific community, however, as this community can expect to welcome students well trained in the methods of science.

EvoS Journal is proud to be among the ranks of undergraduate journals allowing for undergraduate peer review, but also to allow for undergraduates to publish in the same venue as do professionals. In this way, students are initiated into the rigors of academic life in a cordial, constructively critical manner in which they can explore and master evolutionary knowledge. One of the undergraduate reviewers sums it up best by saying, "I would consider submitting a paper to EvoS Journal in the future, because the peer-review evaluation process provides for quality publications, as well as productive learning opportunities for undergraduates new to research."

Acknowledgments Thank you to two anonymous reviewers and the guest editor (Dr. Geher) for constructive comments on earlier drafts of this manuscript.

\section{References}

Dochy F, Segers M, Sluijsmans D. The use of self-, peer and coassessment in higher education: a review. Stud High Educ. 1999;24:331-50.

Dominick PG, Reilly RR, McGourty JW. The effects of peer feedback on team member behavior. Group Organ Management. 1997;22:508-20.

Elgren T, Hensel N. Undergraduate research experiences: synergies between scholarship and teaching. Peer Review. 2006;8:4-7.

Garcia J, Geher G, Crosier B, Saad G, Gambacorta D, Johnsen L, et al. The interdisciplinarity of evolutionary approaches to human behavior: a key to survival in the Ivory Archipelago. Futures (2011).

Guilford WH. Teaching peer review and the process of scientific writing. Adv Physiol Educ. 2001;25:167-75.

\footnotetext{
${ }^{1}$ The author would like to thank an anonymous reviewer for this novel research suggestion.
} 
Liu J, Pysarchik DT, Taylor WW. Peer review in the classroom. Bioscience. 2002;52:824-9.

Lundstrom K, Baker W. To give is better than to review: the benefits of peer review to the reviewer's own writing. J Second Lang Writ. 2009;18:30-43.

McGourty J, Dominick P, Reilly RR. Incorporating student peer review and feedback into the assessment process. Proceedings of the 28th Annual Frontiers in Education Conference, Tempe, AZ. 1998:14-19. Retrieved online: http://fie-conference.org/fie98/.

Muller K. Evolutionary educational psychology: the disparity between how children want to learn and how they are being taught. EvoS Journal: The Journal of the Evolutionary Studies Consortium. 2010;2:12-23.

Newell JA. Using peer review in the undergraduate laboratory. Chemical Engineering Education. 1998;32:194-6. Summer issue.
O’Brien DT, Wilson DS, Hawley PH. "Evolution for Everyone": a course that expands evolutionary theory beyond the biological sciences. Evol Educ Outreach. 2009;2:445-57.

Paulus TM. The effect of peer and teacher feedback on student writing. J Second Lang Writ. 1999;8:265-89.

Peters DP, Ceci SJ. Peer-review practices of psychological journals: the fate of published articles. Behav Brain Sci. 1982;5:187-95.

Sargent ME. Peer response to low stakes writing in a WAC literature classroom. New Dir Teach Learn. 1997;69:41-52.

Silva E, Moreira D. WebCoM: a tool to use peer review to improve student interaction. J Educ Resour Computing. 2003;3:1-14.

Smith Z, Arrow H. Evolutionary perspectives on religion: an overview and synthesis. EvoS Journal: The Journal of the Evolutionary Studies Consortium. 2010;2:48-66. 